\title{
Creating a Buzz around Corporate Reputation with Beeswarm Plots
}

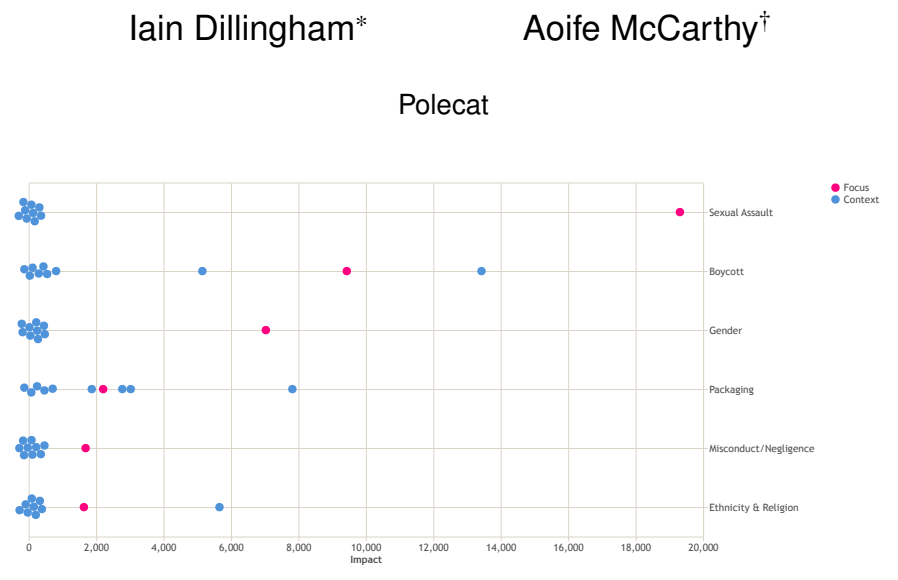

Figure 1: The beeswarm plot answers the question 'How do I compare to my competitors?' without looking 'too statistical.'

\section{INTRODUCTION}

Polecat is a technology company that helps organisations manage their corporate reputation. Polecat's application, called RepVault, processes millions of documents every day to measure the impact associated with roughly 14,000 organisations and 1,500 topics. Impact incorporates the reach and credibility of a document-such as a newspaper article, blog post, or tweet-its relevance to an organisation, and its relevance to a topic.

In RepVault, topics are grouped into taxonomies. There are roughly 200 topics in RepVault's standard taxonomy, such as Autonomous Vehicles, Bitcoin, and Cloud Computing. However, most taxonomies are not standard: they, and their topics, are created specifically for clients.

Typically, clients want to compare themselves to their competitors for some, but not all, topics in a taxonomy. However, we know from experience that clients are uncomfortable with charts that look 'too statistical.' Having considered several charts, we designed a beeswarm plot to show impact by topic for a set of organisations. During testing, responses were positive. Consequently, it will be added to RepVault and introduced to clients.

\section{Why a BEESWARM PLOT?}

Our challenge was to answer the question 'How do I compare to my competitors?' without using charts that look 'too statistical.' Our hypothesis was that clients would be more comfortable if they saw a single mark for a single organisation, as with a dot plot, rather than a single mark for a set of organisations, as with a histogram. However, we know from experience that impact by topic for a set of organisations is often positively skewed. Consequently, if we used single marks for single organisations, then these marks would likely over-plot on a dot plot, making them hard to distinguish.

We were inspired by a beeswarm plot that we saw on the Examples section of the Vega [1] documentation. ${ }^{1}$ The example shows people by group for the well known Les Misérables dataset. The example uses a force transform to pull each node towards a coordinate, whilst also pushing apart nodes whose radii overlap. It is this force

*iain.dillingham@polecat.com

†aoife.mccarthy@polecat.com

${ }^{1}$ https://vega.github.io/vega/examples/beeswarm-plot/ transform that gives the plot the distinctive beeswarm shape. Clearly, a beeswarm plot of impact by topic for a set of organisations ${ }^{2}$ would ensure marks did not over-plot, making them easier to distinguish.

RepVault is designed for the desktop, so we placed topic on the $\mathrm{y}$ axis to allow for long axis labels. We placed impact on the $\mathrm{x}$ axis. We encoded the focus organisation, typically the client, and the context organisations, typically their competitors, using colour.

\section{OUtcome}

We tested the beeswarm plot with six participants: colleagues who interact with clients every day. We adopted a three-stage protocol.

- Teach each participant about the beeswarm plot. We described the $\mathrm{x}$ and $\mathrm{y}$ axes; the colour encoding; and the reason for the distinctive beeswarm shape.

- Test each participant's ability to perform tasks using the beeswarm plot. We asked each participant to estimate the impact associated with the focus organisation and one context organisation, and to describe how the focus organisation is performing with respect to one or more context organisations.

- Discuss each participant's reactions to the beeswarm plot. We asked each participant what questions they would like to ask, and what observations they would like to make, about the beeswarm plot.

We adopted a three-stage protocol because we assumed that participants would be unfamiliar with the beeswarm plot. By teaching each participant about the beeswarm plot, and by moving from closed to open questions when testing, we hoped to encourage informed questions and observations.

Responses were positive: all participants estimated the impact quickly and accurately, and described how the focus organisation is performing fluently. Several participants indicated that the beeswarm plot was a good answer to the question 'How do I compare to my competitors?' Consequently, it will be added to RepVault and introduced to clients.

\section{REFERENCES}

[1] A. Satyanarayan, R. Russell, J. Hoffswell, and J. Heer. Reactive Vega: A Streaming Dataflow Architecture for Declarative Interactive Visualization. IEEE Trans. Visualization \& Comp. Graphics (Proc. InfoVis), 2016.

${ }^{2}$ https://observablehq.com/@iaindillingham/beeswarm-plots 